\title{
Resemblances and differences in mechanisms of noise-induced resonance
}

\author{
R. Centurelli ${ }^{1}$, S. Musacchio ${ }^{1,2}$, R.A. Pasmanter ${ }^{3}$ and A. Vulpiani ${ }^{1,2,4}$ \\ 1 Department of Physics, University La Sapienza, P.le A. Moro 2, I-00185 Roma, Italy. \\ 2 INFM (UdR and CSM) - Unità di Roma La Sapienza. \\ 3 K.N.M.I., P.O.Box 307, 3730 AE, De Bilt, The Netherlands and \\ 4 INFN - Sezione di Roma La Sapienza.
}

(Dated: July 10, 2018)

\begin{abstract}
Systems showing stochastic resonance (SR) or coherent resonance (CR) share some features, in particular the nearby periodic character of the signal. We show that in spite of this resemblance the different underlying dynamics can be detected in experimental data by studying the histogram of inter-spikes times and some statistical properties like two-times correlation functions. We discuss the possible relevance for climate modeling.

PACS numbers: PACS number(s) : 05.10.Gg, 92.60.Ry
\end{abstract}

\section{INTRODUCTION}

The mechanism of stochastic resonance (SR) was initially introduced as a possible explanation of climate changes on long time-scales [1]. During the last two decades it has been applied to a wide class of systems ranging from analog circuits, neurobiology, ring lasers, systems with colored noise, etc; for a review see Ref. [2].

The prototypical system showing SR, which is also the original one used to model climate changes, is the stochastic differential equation

$$
\frac{d x}{d t}=-\frac{\partial V(x, t)}{\partial x}+\sqrt{2 D} \eta,
$$

where $\eta$ is a Gaussian, white noise with $\langle\eta(t)\rangle=0$ and $\left\langle\eta(t) \eta\left(t^{\prime}\right)\right\rangle=\delta\left(t-t^{\prime}\right), D$ measures the noise intensity and $V(x, t)$ a is double well potential with a time periodic term

$$
V(x, t)=\frac{x^{4}}{4}-\frac{x^{2}}{2}+A x \cos (2 \pi t / T) .
$$

In the case of a stationary potential, i.e., $A=0$, the jumps between the two minima at $x=-1$ and $x=1$ are independent events whose probability distribution is approximately Poissonian 3]. Using simple arguments based on the Kramers exit-time formula [4], it can be shown that there is range of values of $D, T$ and $A$ where $\mathrm{SR}$ is present, i.e., the jumps between the two minima (close to -1 and +1 if $A$ is sufficiently small) are strongly synchronized with the forcing and that the probability distribution function (PDF) of the jumping time $\tau$ has a relatively sharp peak around $T$ [1, 2].

The phenomenon of SR provides one example of the nontrivial role that noise can play in dynamical systems with an external periodic forcing. Besides SR, there exist other examples of the "constructive role" of noise, e.g., one can have a synchronization of trajectories generated by different initial conditions and the same noise realization 5]. Our interest will focus on cases where noise can enhance periodic behavior, e.g., the so-called coherent resonance $(\mathrm{CR})$ and the noise-induced dynamics in systems with time delay (ND).

The phenomenon of CR [6] has been found in models describing excitable systems that occur in different fields like chemical reactions, neuronal and other biological processes 7,8 . The prototypical stochastic differential equation used in this case is the FitzHugh-Nagumo system defined by:

$$
\begin{aligned}
\epsilon \frac{d x}{d t} & =x-\frac{x^{3}}{3}-y \\
\frac{d y}{d t} & =x+a+\sqrt{2 D} \eta
\end{aligned}
$$

with $\epsilon \ll 1$ so that the time evolution of $x$ is much faster than that of $y$. For $|a|>1$ there is a stable fixed point, for $|a|<1$ there is an unstable fixed point and a limit cycle. The cycle consists of two pieces of slow motion connected by a fast jump. If $|a|$ is slightly larger than 1 the system is excitable [6], i.e., small deviations from the fixed point may generate large pulses (also called spikes) 9]. Moreover, in this case, one finds that there is a range of values of the noise intensity $D$ such that CR appears, i.e., roughly periodic noise-excited oscillations are present, resembling the SR oscillations [6].

The prototypical example for ND [10] is the overdamped particle motion in the double-well potential 


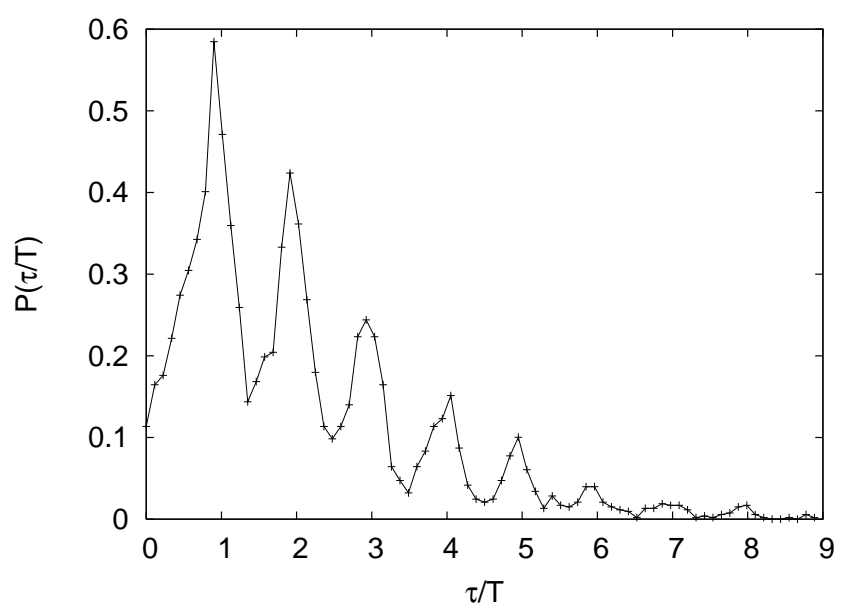

FIG. 1: PDF of the inter-spikes time in the system described by Eq. (11) showing stochastic resonance. The external periodic force has period $T=100$ and amplitude $A=-0.15$. The noise intensity is at the corresponding optimal value $D^{*}=0.10$.

$$
\begin{aligned}
& V(x(t), x(t-T)): \\
& \begin{aligned}
\frac{d x(t)}{d t} & =-\frac{\partial V(x(t), x(t-T))}{\partial x(t)}+\sqrt{2 D} \eta \\
& =x(t)-x(t)^{3}-A x(t-T)+\sqrt{2 D} \eta
\end{aligned}
\end{aligned}
$$

where $T$ is the delay. It is not very difficult to realize that the delay term $A x(t-T)$ has a role similar to that of the periodic forcing in Eq. (1), 21). Accordingly, in a certain range of parameters' values, there is a sort of periodic motion with period $T$ or $2 T$ (this depends upon the sign of $A$ ). This ND equation had been proposed as a model for some climate changes 11].

Although SR, CR and ND, are similar phenomena, in the sense that their time evolution is nearly periodic, there are also some important differences. For example: a) due to the presence of the term $A x(t-T)$, Eq. (5I) is in fact an infinite dimensional system since in order to determine $x(t)$ for $t>0$ one has to specify $x\left(t^{\prime}\right)$ with $-T \leq t^{\prime} \leq 0$. On the contrary for Eq. (11) it is sufficient to know $x(0)$. b) in the case of SR the periodicity is due to the external forcing while in the CR case the periodicity has an internal origin, i.e., the periodic motion is due to the intrinsic dynamics and, at variance with SR, its period cannot be changed by tuning external control parameters. This difference can play an important role in, e.g., the context of climate changes and glaciation, for more details, refer to the last Section.

The aim of this paper is to analyse the differences among SR, ND and CR and their possible relevance to applications. In Sect II we will briefly review some properties of the PDF of the inter-spikes times for SR, ND and

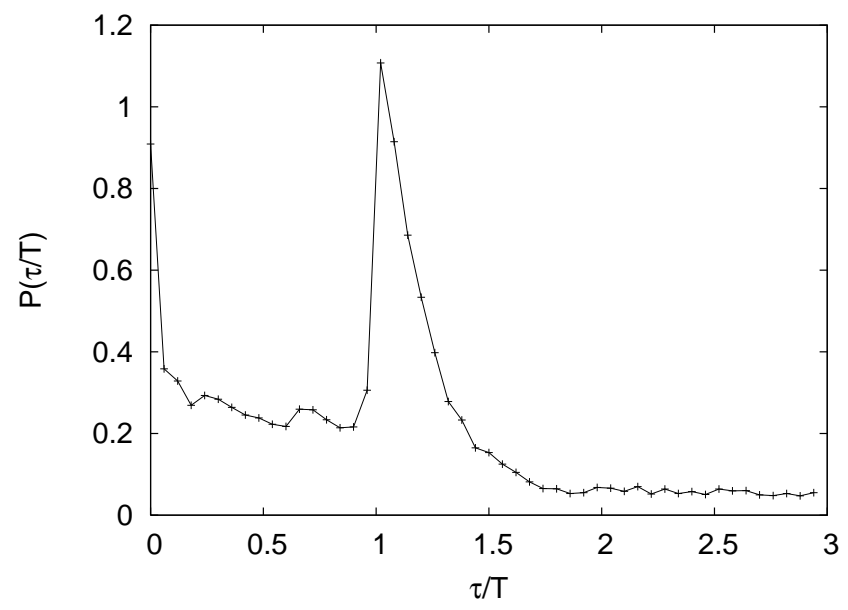

FIG. 2: PDF of the inter-spikes time in the system described

by Eq. (5). The amplitude of the delay term is $A=-0.15$, and the delay time is $T=100$. The noise intensity is at the corresponding optimal value $D^{*}=0.10$.

CR. In particular, we recover a recent result [12] showing that CR and SR are not conflicting or excluding mechanisms, i.e., the same periodically driven system, e.g., the one given by Eqs. (34) with a time-dependent parameter $a(t)=a_{0}+a_{1} \cos (2 \pi t / T)$ can present a transition from SR to CR behavior when the noise intensity $D$ is increased. In Sect III we show that, in spite of some resemblance, SR, ND and CR exhibit different statistical features which, at least in principle, can be detected in experimental data. In particular we have that for SR the correlation function $C(\tau)$, after a transient period, is periodic and does not relax to zero. On the contrary, for the CR and ND cases $C(\tau)$ shows damped oscillations. Sect IV is devoted to general remarks and conclusions. In particular we deal with the potential relevance of the differences between SR, CR and ND to climate modeling.

\section{STATISTICS OF INTER-SPIKES TIMES}

One basic feature shared by the three models introduced in the previous Section, is the presence of two characteristic states: two equilibria in the case of SR and ND, a rest state and an excited one in the FitzHugh-Nagumo model. Jumps between these states are made possible by the noise. Moreover, it turns out that there exists an optimal value of the noise intensity such that this jumping becomes approximately periodic, i.e., the typical time between two consecutive transitions is roughly constant.

According to the terminology of biological systems, where $\mathrm{CR}$ was originally introduced, we will refer to the time interval between consecutive transitions as the 


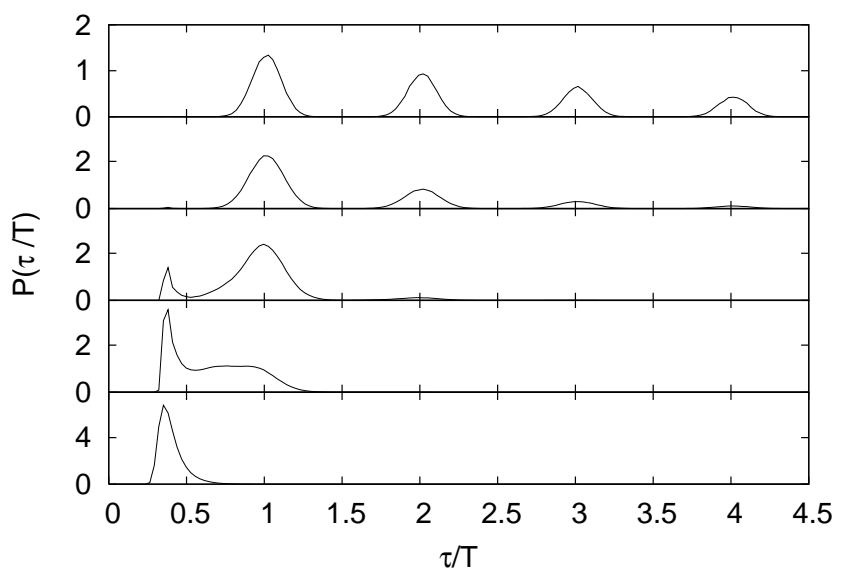

FIG. 3: PDF of the inter-spikes time in the FitzHughNagumo model Eqs. (34) with parameters' values $a_{0}=1.05$, $a_{1}=0.04, \epsilon=0.01$ and $T=10>T_{*}=3.7$ for different noise intensities. From top to bottom, $D=2.5 \times 10^{-5}$, $D=4.3 \times 10^{-5}, D=1.1 \times 10^{-4}, D=2.3 \times 10^{-4}$ and $D=4.3 \times 10^{-3}$.

"inter-spikes time $\tau$ ". In the case of the system (34) where the dynamical variables show well defined maxima, the definition of the inter-spikes time $\tau$ is rather natural. In the case of the other two systems (12) and (15) one can define $\tau$ as $\tau=t_{n+1}-t_{n}$ where $t_{n}$ is the $n$-th crossing time, i.e., $x\left(t_{n}\right)=0$ and $\dot{x}\left(t_{n}\right)>0$. A measure of the signal's periodic character is provided by the normalized variance $N V$ of inter-spikes times, $N V=\sqrt{\operatorname{Var}(\tau)} /\langle\tau\rangle$. For generic noise intensity the transitions occur at random times, and $p(\tau)$, the PDF of inter-spikes times, is weakly localized, i.e., $N V$ is of order 1 . We define the resonant or optimal value of the noise intensity, and denote it by $D^{*}$, as the value of $D$ for which the system has minimal normalized variance $N V$.

In Figure 1 we show the PDF of the inter-spikes time interval in the case of SR for the system described by Eq. (11) at the optimal noise intensity $D^{*}=0.1$. We see that $p(\tau)$ is peaked around $\tau=n T$ with $n=1,2, \ldots .$. , and that the envelope is approximately exponential. This feature can be easily explained as follows. Consider the trajectories $x(t)$, starting at $t=0$ from the favored well, i.e. $x(0)=1$ if $A<0$. In the case of SR, at $t$ close to $T / 4$ many of the trajectories will jump onto the other minimum at $x=-1$ and after half period they will jump back again onto $x=1$. However, a fraction of the trajectories remains in the "wrong position" (i.e. in the unfavored well) for $t$ close to $T$. Calling $P$ the probability of this event, we have that the integral of $p(\tau)$ around $T$, say for $\tau \in[0.5 T, 1.5 T]$, is $(1-P)$. The events with inter-spikes time $\tau \sim n T$ correspond to trajectories $x(t)$ which are in

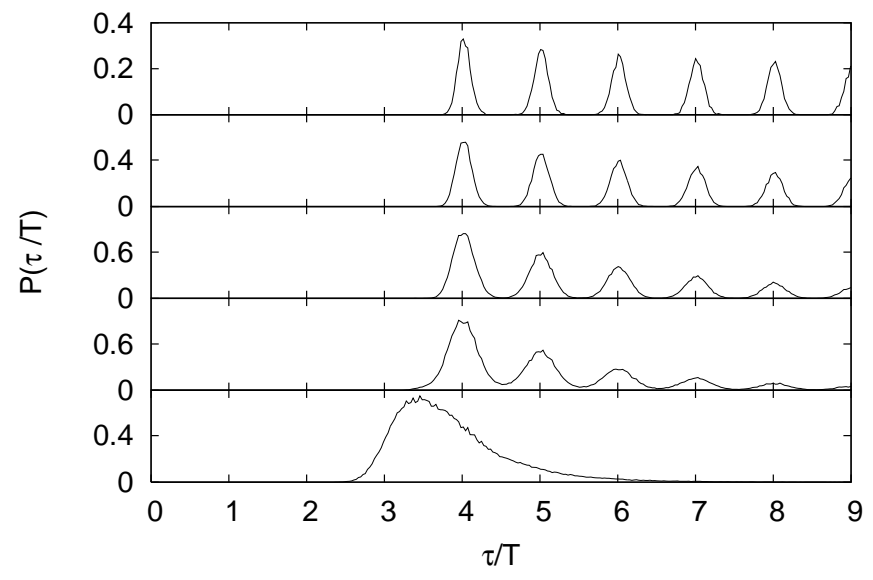

FIG. 4: As in Fig. (3) but with $T=1<T_{*}=3.7$.

the the "wrong" minimum at $T, 2 T, \ldots, n T$. Taking into account the periodicity of the forcing and assuming that the system's memory is much shorter than the external period $T$ we have that the probability to have $x(t)$ in the "wrong" minimum at $t \sim k T$ under the condition that $x(t)$ was in the the "wrong" minimum at $t \sim(k-1) T$, does not depend on the behavior for $t<(k-1) T$. Therefore the integral of $p(\tau)$ around $n T$ is $(1-P) P^{n-1} \sim e^{-c n}$ with $c=-\ln P$. The envelop of the inter-spikes-interval histogram has recently been computed by Berglund and Gentz [13] in a more general and rigorous setting.

In the case of CR (not shown) and ND, see Figure 2 the PDF of the inter-spikes time interval is peaked around $T$ where $T$ is the characteristic internal time of the system, namely the delay time in the ND case, and the period of the limit cycle in the CR case. The parameters of the memory term in the ND-model (5) have been chosen in order to emphasize the similarities with the SR-model, i.e., the amplitude $A$ and delay time $T$ are identical to the amplitude and period of the external forcing in Eq. (1). One consequence of this choice is that the intensity of the optimal noise coincides with that in the SR-model. Notice that, at variance with the SR case, no peaks are present at multiples of $T$.

In summary, in all three systems there exists an optimal noise intensity which produces a roughly periodic signal $x(t)$. At this intensity a sharp peak appears in the inter-spikes time PDF. In the case of CR and ND the PDF has only one maximum at $\tau=T$. On the other hand, in the case of SR other maxima appear at $n T$. This effect can be considered as one of the distinctive marks of the stochastic resonance. In contraposition to the CR and ND cases, the periodicity of the signal in the SR case is induced by an external periodic force, which triggers the jumps at fixed times $t=n T$ in such a way that the system synchronizes with this external "clock".

The combined effects of noise and nonlinearity can re- 


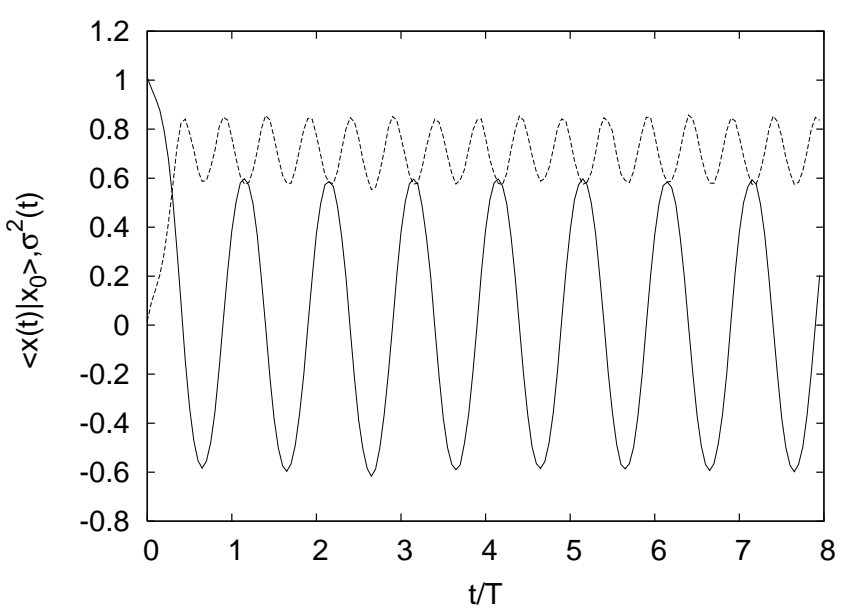

FIG. 5: Conditional average $\left\langle x(t) \mid x_{0}\right\rangle$ (solid line) and conditional variance $\sigma^{2}(t)$ (dashed line) in the case of SR for the system described by Eq. (1). The initial position is $x_{0}=1$, i.e., in the favored well. The external force has period $T=100$ and amplitude $A=-0.15$. The noise intensity is at its optimal level $D=0.10$. The average is taken over $N=10^{4}$ realizations of the noise.

sult in more complex behavior 14, 15]. In particular it has recently been shown in 12] that SR and CR can coexist in the same system. This behavior occurs, e.g., in the model (34) when small oscillations are imposed on the control parameter $a(t)=a_{0}+a_{1} \cos (2 \pi t / T)$. We consider only $a_{0}$ and oscillation amplitudes $a_{1}$ such that the control parameter $a(t)$ never crosses the critical value, i.e. $a(t)>1, \forall t$. The presence of these small oscillations determines privileged times $t_{n}=(2 n+1) T / 2$ at which the system is closer to the excited state, i.e., $a\left(t_{n}\right)$ approaches 1 from above, and a noise induced transition is facilitated. Analogously to the case of the double well system with periodic forcing, there exists an optimal noise intensity for which a regular, quasi-periodic behavior emerges. More precisely: at low noise intensity, $p(\tau)$ has rather sharp peaks at $\tau=n T$ with an approximately exponential envelope, showing all the features of SR, see the two upper panels in Figure 3] At higher noise intensity, as in the two lower panels in Figure 3 the small oscillations in $a(t)$ become irrelevant and the system shows $\mathrm{CR}$ as if the control parameter were fixed at its mean value $a_{0}$. The shape of $p(\tau)$ behaves accordingly as the noise increases: the maxima of SR diminish and a single peak with an exponential tail appears at $\tau=T_{*}$ where $T_{*}$ is the period of the system's limit cycle.

Moreover, when the external force period $T$ is shorter than the internal, limit-cycle period $T_{*}$ there are peaks only for $n T \geq T_{*}$ because once the excited state is reached the system needs at least a time $T_{*}$ in order to

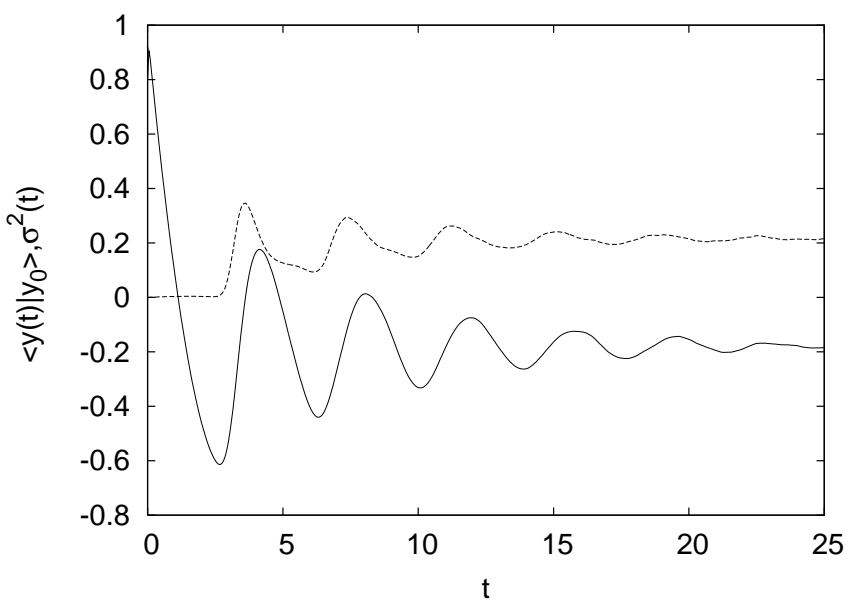

FIG. 6: Same as in Fig. 5 in the case of CR for the system described by Eqs. (34) with $a=1.05, \epsilon=0.01$ and optimal noise intensity $D=2.5 \times 10^{-3}$. The initial position is $x_{0}=$ $2, y_{0}=0.8$, i.e., in the excited state. The average is taken over $N=10^{4}$ realizations of the noise.

relax back into the excitable, rest state and restart the whole cycle (see fig. 4).

\section{CONDITIONAL AVERAGES AND CORRELATION FUNCTIONS}

If one would observe just a single trajectory, SR, CR and ND would appear rather similar since the three cases would present us with a nearly periodic $x(t)$. An analysis based on Fourier spectra, as it is often done, would reinforce this picture. In the previous Section we contrasted the multi-peaked PDF of inter-spikes time in the SR case with the one-peaked PDF in the $\mathrm{CR}$ and $\mathrm{ND}$ cases. In this Section we bring to the fore some statistical properties which are present in the SR case but are absent both in the CR and in the ND cases.

Consider an ensemble of $N$ trajectories $\left\{x^{(n)}(t), n=\right.$ $1, \ldots, N \gg 1\}$, sharing the same initial conditions $x^{(n)}(0)=x_{0}$, but with different realizations of the noise $\eta(t)$ and compute from Eqs. (1), (3) 4) and (5) the conditional average $\left\langle x(t) \mid x_{0}\right\rangle$,

$$
\left\langle x(t) \mid x_{0}\right\rangle=\frac{1}{N} \sum_{n=1}^{N} x^{(n)}(t),
$$

and the conditional variance

$$
\begin{aligned}
\sigma^{2}(t) & =\left\langle x^{2}(t) \mid x_{0}\right\rangle-\left\langle x(t) \mid x_{0}\right\rangle^{2} \\
& =\frac{1}{N} \sum_{n=1}^{N}\left[x^{(n)}(t)-\left\langle x(t) \mid x_{0}\right\rangle\right]^{2},
\end{aligned}
$$




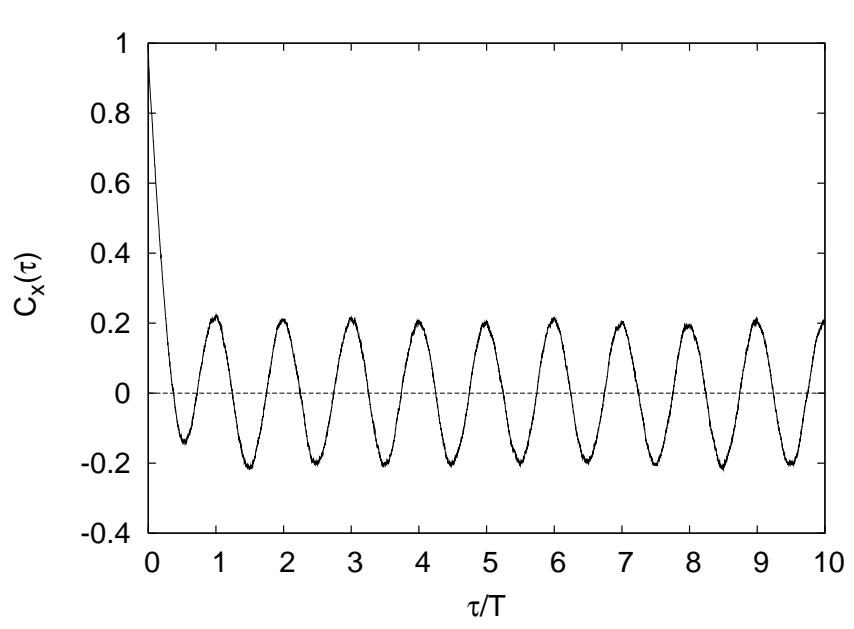

FIG. 7: The periodic correlation function $C(\tau)$ of the SR model Eq. (1). Parameters' values as in Fig. 1]

In Figs. 5 and 6 we show $\left\langle x(t) \mid x_{0}\right\rangle$ and $\sigma^{2}(t)$ as functions of the time $t$, for the SR case and the CR case respectively. In both cases the noise-intensity values $D$ are the optimal ones. In the SR case $\left\langle x(t) \mid x_{0}\right\rangle$ does not relax to zero at large times $t$ and, ignoring the initial transient, it is periodic. The conditional variance $\sigma^{2}(t)$ reaches its minima when the absolute value of the conditional average $\left\langle x(t) \mid x_{0}\right\rangle$ reaches its maxima. The largest values for $\sigma^{2}(t)$ are achieved when $\left\langle x(t) \mid x_{0}\right\rangle$ is around zero. In other words, the main uncertainty in the process occurs around $T / 4,3 T / 4,5 T / 4$ and so on, i.e., when the jumps between the two minima take place.

As it can be seen in Fig 6 the behavior found in the CR case is different. Even with the noise intensity at its optimal value, after a few damped oscillations both the conditional average and variance relax to the constant values $\langle\langle x(t)\rangle\rangle$ and $\left\langle\left\langle x^{2}(t)\right\rangle\right\rangle-\langle\langle x(t)\rangle\rangle^{2}$, where $\langle\langle\cdots\rangle\rangle$ indicates a time average.

This behavior underlines the intrinsic difference between the SR and CR mechanisms. In the case of SR, the presence of an external synchronizing force, makes the transitions from state +1 to state -1 to occur around preferred times. A set of independent replicas, initially localized in one of the two wells, will therefore quickly reach a periodic configuration, with the maximum probability localized in the time-dependent favored well. On the contrary, in the CR case, there are no externally defined preferred times for the transitions, therefore each replica quickly loses its initial synchronization with the other ones and after a few periods the jumps occur at different times for different replicas.

The correlation function

$$
C(\tau)=\frac{\langle\langle x(t+\tau) x(t)\rangle\rangle-\langle\langle x\rangle\rangle^{2}}{\left\langle\left\langle x^{2}\right\rangle\right\rangle-\langle\langle x\rangle\rangle^{2}}
$$

behaves similarly to $\left\langle x(t) \mid x_{0}\right\rangle$ : in the $\mathrm{CR}$ case it relaxes

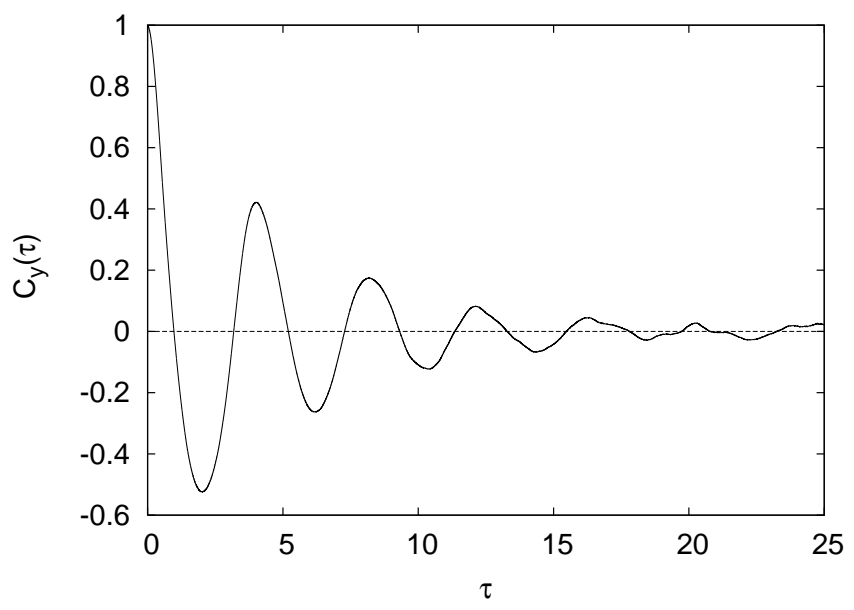

FIG. 8: Correlation function $C(\tau)$ of the $y$ variable for the FitzHugh-Nagumo model described by Eqs. (3] , 4). Parameters' values as in Fig. [6]

to zero while in the SR case it remains periodic with non decreasing amplitude, see Figs. (7) and (8). If one defines a correlation time $\tau_{c}$ as

$$
\tau_{c}=\int_{0}^{\infty} C(\tau)^{2} d \tau
$$

one finds that $\tau_{c}$ diverges in the $\mathrm{SR}$ case while it remains finite in the CR case and that, as a function of the noise intensity, it attains its maximum $\tau_{c}=1.03$ at the optimal noise intensity value. The behavior in the ND case (not shown) is qualitatively very similar to that obtained in the CR case.

\section{CONCLUSIONS AND DISCUSSION}

The numerical study of SR, CR and ND presented in this paper is focused on contrasting statistical features which would be difficult or impossible to detect by simply looking at the spectrum since the spectrum shows a peak that achieves a maximal sharpness for the optimally chosen noise intensities in all the cases we have studied. As we have seen, it is possible to distinguish between the different underlaying dynamical mechanisms by studying the PDF of inter-spikes times, conditional averages and time-delayed correlation functions.

We believe that these results are of interest not only in the context of dynamical systems but also in the study of certain climate phenomena. For time scales of order $O\left(10^{5} \mathrm{ys}\right)$ and larger, Milankovich [16] has proposed that Earth's climate has been determined by the influx of solar energy to such an extent that the fluctuations in, e.g., the global mean temperature and seasonality must have 
been closely correlated with the variations in the incoming energy flux due to the periodicities in Earth's orbit.

Another possible issue, for which the presented results are potentially interesting, is the so-called DansgaardOeschger(DO) events [17] which have been inferred from the study of Late-Pleistocene ice cores and marine sediments. These measurements show rapid warmings of the atmosphere followed by a much slower decay back into the average glacial conditions. The warmings took place on a time scale of a few decades while the relaxation back into glacial temperatures lasted some centuries up to millennia. They seem to have occurred at intervals of $1.500 \pm 200$ years or integer multiples hereof [18]. They were absent during the Holocene, i.e., during the $10^{4}$ years before present. The discovery of these rapid warmings led to proposing a number of possible explanations, some of them favoring the internal origin of the period approximately equal to 1.500 years [19, 20, 21], while other explanations assume a similar period due to an external astronomical forcing 22, i.e. a SR-type scenario. In particular, it was shown that in an ocean-circulation box model and within an appropriate parameters' range, the purely deterministic system has a fixed point and does not show any time dependence while the addition of noise leads to the generation of spikes with a well defined inter-spikes time interval [23], i.e., that coherence resonance is present in this ocean circulation model. As discussed in Section II, now we know that, at least in some systems, it is possible to observe either CR or SR behavior depending upon the noise intensity.

We have shown that it is possible to distinguish between the SR and CR, e.g., by looking at time-delayed correlation functions and at the PDF of the inter-spikes times. Needless to say, in order to compute such correlation functions, or the PDF, a sufficiently long and accurate series of measurements is required. From the limited information about the DO events that has been extracted from the geological record it is difficult to decide in favor of one scenario or the other. Indeed, the $\mathrm{PDF}$ for the inter-spikes time is qualitatively in agreement with the one observed in the SR case. On the other hand the time values at which one observes peaks of the PDF do not correspond to known astronomical periods, accordingly, a CR scenario would seem more appropriate.

\section{Acknowledgments}

We thank A. Timmermann and A.S. Pikovsky for stimulating discussions and useful comments. This work has been supported by MIUR-COFIN03 "Complex Systems and Many-Body Problems" (2003020230) and the INFM (Statistical Mechanics and Complexity Center, Rome).
[1] R. Benzi, A. Sutera and A. Vulpiani, J. Phys. A 14, L453 (1981); C.Nicolis Tellus 34, 1 (1982); R. Benzi, G. Parisi, A. Sutera and A. Vulpiani, Tellus 34, 10 (1982); R. Benzi, G. Parisi, A. Sutera and A. Vulpiani, SIAM J. Appl Math 43, 565 (1983).

[2] K. Wiesenfeld and F. Moss, Nature 373,33 (1995); L. Gammaitoni, P. Hänggi, P. Jung, and F. Marchesoni, Rev. Mod. Phys. 70, 223 (1998).

[3] C. W. Gardiner Handbook of Stochastic Methods (Springer-Verlag, Berlin 1990)

[4] S. Chandrasekhar, Rev. Mod. Phys. 15, 1 (1943).

[5] K. Matsumoto and I. Tsuda, J. Stat. Phys. 31, 87 (1983).

[6] A.S. Pikovsky and J. Kurths, Phys. Rev. Lett. 78, 775 (1997).

[7] X. Pei, K. Bachmann and F. Moss, Phys. Lett. A 206, 61 (1995).

[8] J.J. Collins, C.C. Chow and T.T. Imhoff, Phys. Rev. E 52, R3321 (1995).

[9] A.C. Scott Rev. Mod. Phys. 47,487 (1975).
[10] L.S. Tsimring and A. Pikovsky, Phys. Rev. Lett. 87, 250602 (2001).

[11] J. P. Pelletier, J. Geophys. Res. Atmos. 108 (D20), 4645 (2003).

[12] GerardoJ. Escalera Santos, M. Rivera, P. Parmananda, Phys. Rev. Lett. 92, 230601 (2004).

[13] N. Berglund and B. Gentz, http://www.arxiv.org/abs/cond-mat/0408321 (2004).

[14] A. M. Yacomotti, M. C. Eguia, J. Aliaga, O. E. Martinez, G. B. Mindlin and A. Lipsich, Phys. Rev. Lett. 83, 292 (1999).

[15] J. M. Mendez, J. Aliaga and G. B. Mindlin, Phys. Rev. Lett. 89, 160601 (2002).

[16] W. S. Broecker et al., Science 159, 297-300 (1968); R. A. Kerr, Science 235, 973 (1987).

[17] Dansgaard W., S. J. Johnson, H. B. Clauser, D. DahlJensen, N. Hammer and C. U. Oeschger, (1984), Geophys. Monogr. no. 29, Amer. Geophys. Union, 288-298.

[18] M. Schulz, Paleoceanogr. 17, doi:10.1029/2000PA000571 
(2002).

[19] W. S. Broecker et al., Paleoceanogr. 5, 469, (1990)

[20] M. Winton, in Ice in the climate system, pp. 417, ed. W. R. Peltier, (Springer-Verlag, Berlin 1993).

[21] K. Sakai and W. R. Peltier, J. Climate 10, 949 (1997).

[22] A. Ganopolski, and Rahmstorf, Nature 409, 153 (2001).
[23] Schulz M., A. Paul and A. Timmermann (2002), Geophys. Res. Letters, 29, doi:10.1029/2002GL016144; Timmermann A., H. Gildor and E. Tziperman, (2003), J. Climate, 16, 2569 - 2585. 